\title{
Characteristics of Japanese Patients with Chronic Gastritis and Comparison with Functional Dyspepsia Defined by ROME III Criteria: Based on the Large-Scale Survey, FUTURE Study
}

\author{
Yoshikazu Kinoshita ${ }^{1}$, Tsutomu Chiba $^{2}$ and The FUTURE Study Group
}

\begin{abstract}
Objective To clarify the clinical characteristics of patients diagnosed with chronic gastritis in Japan, a large scale clinical survey of patients with chronic upper gastrointestinal symptoms was conducted. Patients diagnosed with functional dyspepsia (FD) according to the ROME III criteria were selected from among patients with chronic gastritis and the clinical characteristics of patients with FD and those with chronic gastritis were compared.

Patients and Methods Patients with upper abdominal symptoms and diagnosed with chronic gastritis were enrolled in the study. Their main complaints, duration of symptoms, clinical characteristics, mental state, and results of endoscopic examinations, if available, were prospectively surveyed. Quality of life (QOL) impairment caused by abdominal symptoms was also surveyed using the Izumo QOL scale.

Results A total of 9,125 patients with a clinical diagnosis of chronic gastritis were enrolled in the survey. Of those, approximately $60 \%$ had more than 2 symptoms and QOL impairment was greater in cases with multiple symptoms. Endoscopic examinations were performed in 2,946 cases (32.3\% of enrolled patients), during which gastric and/or esophageal carcinoma was found in only $0.2 \%$, though organic diseases were found in $6.2 \%$. Endoscopic examinations were not done for patients with high risks of organic diseases. Of patients with organic diseases excluded by an endoscopic examination, only 362 (12.3\% of patients who underwent an endoscopy) were diagnosed with FD according to the ROME III criteria, mainly because of short symptom duration. There were no remarkable differences in regard to clinical characteristics, including symptoms and mental state, between patients with chronic gastritis and those with FD.

Conclusion Clinical characteristics of patients with chronic gastritis were similar to those with FD, except for shorter symptom duration.
\end{abstract}

Key words: epigastralgia, epigastric fullness, symptom, Izumo scale, endoscopy

(Intern Med 50: 2269-2276, 2011)

(DOI: 10.2169/internalmedicine.50.5678)

\section{Introduction}

In Japan, the term chronic gastritis has been used to describe patients with chronic upper abdominal symptoms irrespective of gastric mucosal inflammation, as confirmed by endoscopic or pathological findings. However, in western countries, functional dyspepsia (FD) has been defined and is used to describe patients with upper abdominal symptoms $(1,2)$. For the diagnosis of FD, exclusion of organic upper gastrointestinal diseases such as ulcers and cancer is considered to be necessary. Furthermore, according to the recently revised definition (ROME III criteria) $(3,4)$, the absence of organic diseases must be confirmed by an endoscopic examination. In addition, patients with FD must have at least one of the following 4 symptoms for the previous 3

${ }^{1}$ Department of Gastroenterology and Hepatology, Shimane University School of Medicine, Japan and ${ }^{2}$ Gastroenterology and Hepatology, Graduate School of Medicine, Kyoto University, Japan

Received for publication April 21, 2011; Accepted for publication July 6, 2011

Correspondence to Dr. Yoshikazu Kinoshita, kinosita@med.shimane-u.ac.jp 
months with symptom onset at least 6 months prior to diagnosis; post-prandial fullness, early satiation, epigastralgia, and epigastric burning. Patients with post-prandial fullness or early satiation are further defined as post-prandial distress syndrome (PDS)-type FD, while those with epigastralgia or epigastric burning are defined as epigastric pain syndrome (EPS)-type FD.

Although an endoscopic examination is considered necessary for patients with chronic abdominal symptoms to investigate the possible presence of organic diseases, only a portion of such patients actually undergo endoscopy in clinical practice in Japan. Patients report various kinds of abdominal symptoms including nausea and upper abdominal discomfort (5-7), in addition to the 4 typical upper abdominal symptoms defined by the ROME III criteria. In addition, the majority of patients consult a physician as early as within 1 month after the appearance of symptoms in Japan (8), where the national medical insurance program enables easy access to physicians, and they do not generally wait 6 months, one of the criteria. Therefore, a diagnosis of FD based on ROME III criteria is difficult in Japan and the majority of patients with abdominal symptoms are diagnosed with chronic gastritis. As a result, data concerning the similarities and differences between FD and chronic gastritis patients are important for applying effective treatment for FD to Japanese patients with chronic gastritis.

We performed a large scale survey to clarify the clinical characteristics of approximately 10,000 patients clinically diagnosed with chronic gastritis. In addition, the ratio of patients who could also be diagnosed as FD according to the Rome III criteria among the surveyed patients with chronic gastritis was determined. Finally, clinical similarities between chronic gastritis and FD were investigated.

\section{Materials and Methods}

\section{Subjects}

The survey was conducted in 1,063 hospitals and clinics between February and December 2009. Patients with a clinical diagnosis of chronic gastritis approved by the Japanese medical insurance were enrolled. For the enrollment, patients need to have chronic upper abdominal symptoms, although the type and duration of symptoms is not of concern. Endoscopic, pathological and/or laboratory tests to confirm the presence of histological gastritis and the absence of organic abdominal diseases are not required for the enrollment. Those who took a histamine $\mathrm{H}_{2}$ receptor antagonist $\left(\mathrm{H}_{2} \mathrm{RA}\right)$ or proton pump inhibitor (PPI) within 2 weeks of the study were excluded.

The following information was obtained from the enrolled patients: main abdominal symptoms, duration of symptoms, and clinical characteristics including gender, age, body mass index (BMI), mental health, habitual use of alcoholic beverage, smoking habit, and consumption of caffeine rich drinks. Abdominal symptom-related QOL impairment was assessed by the Izumo scale questionnaire (9-12). The intensity of abdominal symptoms was assessed by the face scale. Helicobacter pylori $(H$. pylori) infection status and the results of endoscopic examinations were also collected when available. The present study was conducted in compliance with the Good Post-Marketing Study Practice (GPSP) guidelines, the standard for implementation of a post-marketing survey of drugs issued by a regulatory authority in Japan.

\section{Izumo scale questionnaire}

The Izumo scale questionnaire was used to assess abdominal symptom-related impairment of QOL in the enrolled patients. This scale is an abdominal symptom-specific QOL questionnaire that includes 15 items in 5 domains: Reflux, Pain, Fullness, Constipation, and Diarrhea, with 3 items in each domain. The patients were asked to respond to each question using the 6-point Likert Scale, from not disturbed at all (score 0) to intolerable (score 5).

The sum of the scores obtained in 3 questions for each type of abdominal symptom was calculated to determine specific abdominal symptom-related QOL impairment in the categories of heartburn, epigastralgia, and epigastric fullness. Upper abdominal symptom-related QOL impairment was determined as the sum of the heartburn, epigastralgia, and epigastric fullness-related QOL impairment scores. Scores for diarrhea- and constipation-related QOL were not evaluated in this study, since we focused on patients with upper abdominal symptoms. Patients who reported a score of 3 (disturbed) or higher to at least 1 question concerning upper abdominal symptoms were considered to have impaired QOL.

\section{Face scale questionnaire}

The face scale, which was used to assess the intensity of three symptoms (heartburn, epigastralgia, and epigastric fullness), was scored on a 5-point scale from "no distress (0 point)" to "maximum imaginable distress (4 points)". The scores were analyzed for each symptom.

\section{Statistical analysis}

Nominal data were described as frequency and proportion, and numerical data were described as the mean and standard deviation. For comparisons of differences between groups for numerical data, a Wilcoxon rank sum test or unpaired ttest was used. A Jonckheere trend test was used for trends across 3 categories for "duration of chronic disease." For nominal data, Fisher's exact test was used. $\mathrm{p}<0.05$ was considered to indicate statistical significance for all tests.

\section{Results}

\section{Patients}

Small hospitals with fewer than 200 beds and clinics comprising $90.9 \%$ of the institutions where the study was conducted, while $83.3 \%$ of the participating institutions were not teaching or referral centers for gastrointestinal diseases. 


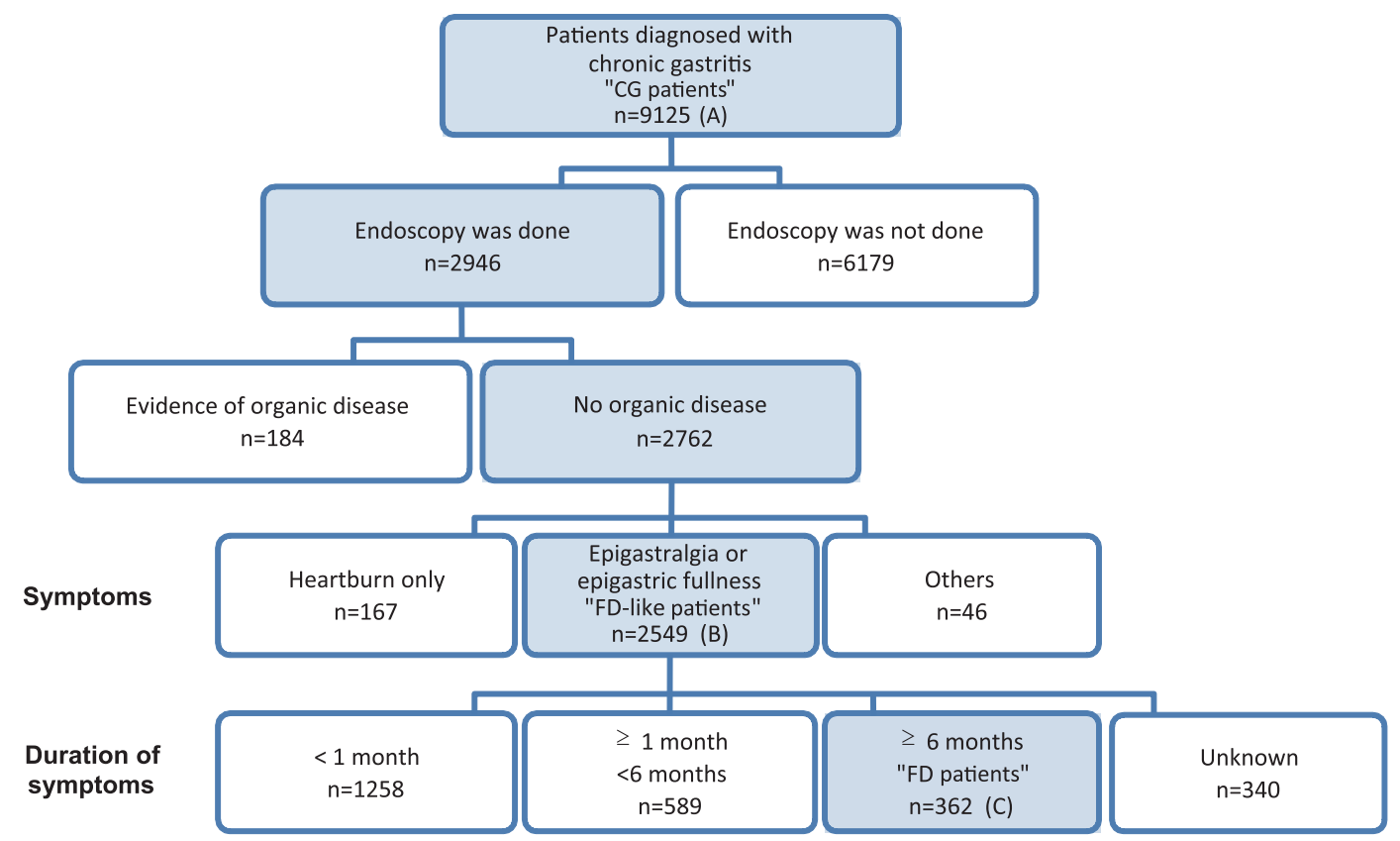

Figure 1. Patients enrolled in this study. Of 9,125 patients with a clinical diagnosis of chronic gastritis, 362 were diagnosed with FD according to the Rome III criteria. (A) Patients diagnosed with chronic gastritis (CG patients). (B) Patients with FD-like symptoms (FD-like patients). (C) Patients with FD (FD patients).

The majority of the enrolled patients were diagnosed in private clinics of primary care physicians and no referred patient was included in this study. Therefore, this study was considered to be conducted mainly by primary care physicians in clinics.

Of all enrolled patients $(n=9,125)$ with a clinical diagnosis of chronic gastritis, 2,946 (32.3\%) underwent endoscopic examinations (Fig. 1). Of the 2,762 patients without endoscopy-detectable organic diseases, 2,549 had at least 1 of the 4 abdominal symptoms required for diagnosis of FD. Of those, only 362 had symptoms for more than 6 months and were finally diagnosed with FD according to the ROME III criteria. Thus, in our population living in Japan, fewer than $5 \%$ of patients with a clinical diagnosis of chronic gastritis could also be diagnosed with FD, due to the infrequent use of endoscopic examinations and shorter duration of abdominal symptoms.

\section{Endoscopic examinations}

To explore the indications for selecting patients for an endoscopic examination used in clinical practice, the clinical characteristics of patients who did and did not undergo endoscopy were compared (Table 1). Endoscopy was used more frequently in females, elderly, patients who did not use low dose aspirin (LDA) or non-steroidal anti-inflammation drugs (NSAIDs), those with shorter duration of symptoms, and those with lighter intensity of symptoms. On the other hand, endoscopy-proven organic diseases were more frequently found in males, elderly, patients with obesity (BMI $>25$ ), and patients with impaired QOL caused by heartburn (Table 2). The clinical characteristics of the patients who need an endoscopy examination because of a higher risk of organic diseases did not fit those of endoscopy investigated cases.

Organic diseases were found in 184 patients $(6.2 \%$ of endoscopy investigated cases). Reflux esophagitis was the most frequently found organic disease, followed by peptic ulcers, as shown in Table 3. Reflux esophagitis was diagnosed endoscopically as the cases with mucosal breaks at the distal esophagus. All of the patients with mucosal breaks including Los Angeles classification grade A-D were diagnosed as cases with reflux esophagitis (12). One patient with esophageal cancer and 5 with gastric cancer were found among 2,946 investigated cases with the clinical diagnosis of chronic gastritis.

\section{Clinical characteristics of patients with chronic gas- tritis, FD and FD-like}

The characteristics of patients with a clinical diagnosis of chronic gastritis were compared with those of FD based on the ROME III criteria and those with FD-like symptoms caused not by endoscopy-proven organic diseases. According to the criteria, patients were defined as FD if a patient had symptoms for over 6 months and did not have heartburn as the only symptom. There were strong similarities in regard to gender, age, BMI, and symptom-related QOL impairment among patients with chronic gastritis and those with FD or FD-like group (Table 4, Fig. 1).

In patients without organic diseases, QOL impairment and the intensity of symptoms were compared between patients with FD and patients with FD symptoms but with a shorter duration, as shown in Fig. 2 and 3. Epigastralgia-related 
Table 1. Differences between Patients who Did and Did Not Undergo Endoscopy

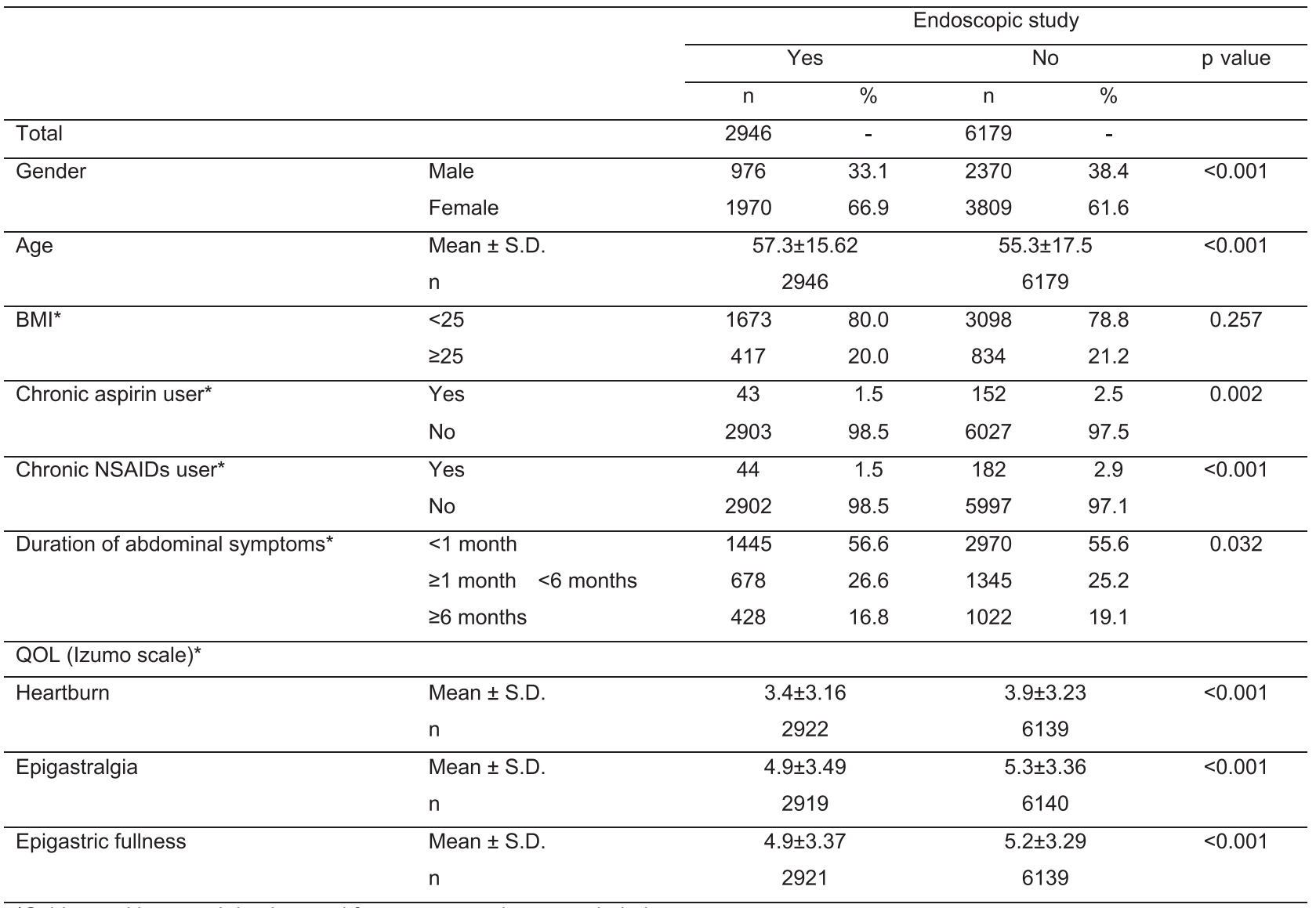

*Subjects without each background factor measured were excluded.

QOL was similarly significantly impaired regardless of the duration of symptoms. Epigastric fullness-related QOL was also significantly impaired, even in patients with symptom duration less than 1 month. Thus, abdominal symptomrelated QOL was found to be significantly impaired as early as 1 month after the appearance of symptoms in patients with chronic gastritis. The intensity of symptoms of epigastralgia and epigastric fullness were similarly strong regardless of the duration of symptoms.

\section{Number of symptoms and QOL impairment}

Abdominal symptom-related QOL impairment was evaluated in patients with different types and numbers of symptoms (Fig. 4). In both patients with chronic gastritis and those with FD, all 3 types (heartburn-related, epigastralgiarelated, epigastric fullness-related) of QOL impairment were found to be more prevalent in patients with multiple symptoms. Therefore, abdominal symptoms were considered to augment QOL impairment by interacting with each another.

\section{Discussion}

We conducted a large scale survey to clarify the present standard clinical situation in Japan regarding patients with chronic gastritis. Two-thirds of the enrolled patients with chronic gastritis were not investigated by endoscopy and therefore diagnosed based on symptoms. Organic diseases were found in only $6.2 \%$ of the endoscopy investigated cases in this study. According to a previous publication in Japan, organic diseases were found in $26.7 \%$ of investigated patients with chronic upper abdominal symptoms (14). In western countries, an endoscopic examination was reported to detect peptic ulcers, reflux esophagitis, and cancer in 15$25 \%, 5-15 \%$, and less than $2 \%$, respectively, of patients with dyspepsia (15). The prevalence of organic diseases in this study was lower than in previous publications, which might be related to the inclusion criteria used in this study and to the inappropriate use of endoscopic examination in clinical practice for patients with upper abdominal symptoms in Japan. First, the inclusion criteria in this study included the cases diagnosed as chronic gastritis because of the chronic upper abdominal symptoms. Therefore, different from previous publications, not all of the abdominal symptomatic patients who visited outpatient clinics were enrolled. Cases who could be clinically diagnosed as organic diseases were excluded from this study. This will decrease the prevalence of organic diseases in this study. Secondly, endoscopic studies were not appropriately done on the high risk patients who were expected to have higher complication rates of organic diseases. Patients over the age of 55, and those treated with NSAIDs or LDA are known to have a higher risk for organic diseases, such as peptic ulcers and reflux esophagi- 
Table 2. Differences between Patients with and without Endoscopy Proven Organic Disease

\begin{tabular}{|c|c|c|c|c|c|c|}
\hline & & \multicolumn{5}{|c|}{ Organic disease } \\
\hline & & \multicolumn{2}{|c|}{ Found } & \multicolumn{2}{|c|}{ Not found } & \multirow[t]{2}{*}{$p$ value } \\
\hline & & $\mathrm{n}$ & $\%$ & $\mathrm{n}$ & $\%$ & \\
\hline Total & & 184 & - & 2762 & - & \\
\hline \multirow[t]{2}{*}{ Gender } & Male & 75 & 40.8 & 901 & 32.6 & 0.029 \\
\hline & Female & 109 & 59.2 & 1861 & 67.4 & \\
\hline \multirow[t]{2}{*}{ Age } & Mean \pm S.D. & \multicolumn{2}{|c|}{$61.3 \pm 14.25$} & \multicolumn{2}{|c|}{$57.1 \pm 15.67$} & $<0.001$ \\
\hline & $\mathrm{n}$ & \multicolumn{2}{|c|}{184} & \multicolumn{2}{|c|}{2762} & \\
\hline \multirow[t]{2}{*}{$\mathrm{BMI}^{*}$} & $<25$ & 81 & 62.8 & 1592 & 81.2 & $<0.001$ \\
\hline & $\geq 25$ & 48 & 37.2 & 369 & 18.8 & \\
\hline \multirow[t]{2}{*}{ Chronic aspirin user* } & Yes & 4 & 2.2 & 39 & 1.4 & 0.341 \\
\hline & No & 180 & 97.8 & 2723 & 98.6 & \\
\hline \multirow[t]{2}{*}{ Chronic NSAID user* } & Yes & 6 & 3.3 & 38 & 1.4 & 0.053 \\
\hline & No & 178 & 96.7 & 2724 & 98.6 & \\
\hline \multirow[t]{3}{*}{ Duration of abdominal symptoms* } & $<1$ month & 89 & 53.6 & 1356 & 56.9 & 0.087 \\
\hline & $\geq 1$ month $<6$ months & 39 & 23.5 & 639 & 26.8 & \\
\hline & $\geq 6$ months & 38 & 22.9 & 390 & 16.4 & \\
\hline \multicolumn{7}{|l|}{ QOL (Izumo scale)* } \\
\hline \multirow[t]{2}{*}{ Heartburn } & Mean \pm S.D. & \multicolumn{2}{|c|}{$4.4 \pm 3.28$} & \multicolumn{2}{|c|}{$3.4 \pm 3.14$} & $<0.001$ \\
\hline & $\mathrm{n}$ & \multicolumn{2}{|c|}{183} & \multicolumn{2}{|c|}{2739} & \\
\hline \multirow[t]{2}{*}{ Epigastralgia } & Mean \pm S.D. & \multicolumn{2}{|c|}{$4.9 \pm 3.67$} & \multicolumn{2}{|c|}{$4.9 \pm 3.48$} & 0.589 \\
\hline & $\mathrm{n}$ & \multicolumn{2}{|c|}{182} & \multicolumn{2}{|c|}{2737} & \\
\hline \multirow[t]{2}{*}{ Epigastric fullness } & Mean \pm S.D. & \multicolumn{2}{|c|}{$4.7 \pm 3.46$} & \multicolumn{2}{|c|}{$4.9 \pm 3.37$} & 0.831 \\
\hline & $\mathrm{n}$ & \multicolumn{2}{|c|}{183} & \multicolumn{2}{|c|}{2738} & \\
\hline
\end{tabular}

*Subjects without each background factor measured were excluded.

Table 3. Organic Diseases Found during Endoscopic Study

\begin{tabular}{lcc}
\hline & $\mathrm{n}$ & $\%$ \\
\hline Patients investigated by endoscopy & 2946 & 100 \\
\hline No organic disease & 2762 & 93.8 \\
Organic diseases & 184 & 6.2 \\
\hline Reflux esophagitis & 143 & 4.8 \\
\hline Peptic ulcer & 40 & 1.4 \\
\hline gastric ulcer & 14 & \\
duodenal ulcer & 27 & \\
\hline Acute gastric mucosal lesion & 1 & \\
\hline Cancer & 6 & 0.2 \\
esophageal cancer & 1 & \\
gastric cancer & 5 & \\
\hline
\end{tabular}

tis (16-19). In addition, older patients have a higher risk of developing esophago-gastric cancer $(20,21)$. In the present study, the majority of enrolled patients over 55 years old were not investigated by endoscopy, and endoscopic examinations were performed less frequently in patients treated by NSAIDs or aspirin. A possible explanation is the already regularly performed endoscopic screening examinations on those high risk patients independent of this study. Because of the recently performed endoscopic examinations, the physicians may skip the endoscopic study in high risk patients. Anyway, based on our findings, the appropriate indications for an endoscopic examination are considered to be established and should be explained to all physicians, including primary care providers, who may participate in the treatment of patients with chronic gastritis in Japan.

In all of the present endoscopy investigated patients with a clinical diagnosis of chronic gastritis, only $12.3 \%$ could be diagnosed with FD according to the ROME III criteria. In this study, because of the lack of information concerning the patients who had 3 months of continuing symptoms that started over 6 months previously, patients who had 6 months of continuing symptoms were diagnosed as FD that is applicable to the ROME III criteria. The necessary 6-month duration of upper abdominal symptoms was the strongest limiting factor for that diagnosis. Over $80 \%$ of our endoscopy investigated patients with FD symptoms had a duration of symptoms of less than 6 months. A possible reason why the 6-month symptom period was included for the diagnosis of FD in ROME III is that progressive diseases such as cancer can easily be excluded during a 6-month observation period. However, in Japan, probably because of easy access to medical care, the majority of dyspeptic patients consult phy- 
Table 4. Clinical Characteristics of Enrolled Patients

\begin{tabular}{|c|c|c|c|c|c|c|c|c|}
\hline & & & \multicolumn{2}{|c|}{ (A) CG patients } & \multicolumn{2}{|c|}{ (B)FD-like patients } & \multicolumn{2}{|c|}{ (C)FD patients } \\
\hline & & & $\mathrm{n}$ & $\%$ & $\mathrm{n}$ & $\%$ & $\mathrm{n}$ & $\%$ \\
\hline \multicolumn{3}{|l|}{ Total } & 9125 & - & 2549 & - & 362 & - \\
\hline \multirow[t]{2}{*}{ Gender } & \multicolumn{2}{|l|}{ Male } & 3346 & 36.7 & 827 & 32.4 & 125 & 34.5 \\
\hline & \multicolumn{2}{|l|}{ Female } & 5779 & 63.3 & 1722 & 67.6 & 237 & 65.5 \\
\hline Age & \multicolumn{2}{|l|}{ Mean \pm S.D. } & \multicolumn{2}{|c|}{$56.0 \pm 17.0$} & \multicolumn{2}{|c|}{$56.6 \pm 15.7$} & \multicolumn{2}{|c|}{$57.1 \pm 15.6$} \\
\hline \multirow[t]{2}{*}{$\mathrm{BMI}^{*}$} & \multicolumn{2}{|l|}{$<25$} & 4771 & 79.2 & 1486 & 81.8 & 223 & 79.6 \\
\hline & \multicolumn{2}{|l|}{$\geq 25$} & 1251 & 20.8 & 331 & 18.2 & 57 & 20.4 \\
\hline Psychological symptoms like & \multicolumn{2}{|l|}{ Yes } & 1616 & 19.6 & 443 & 19.1 & 80 & 23.7 \\
\hline depression and anxiety* & \multicolumn{2}{|l|}{ No } & 6610 & 80.4 & 1881 & 80.9 & 257 & 76.3 \\
\hline \multirow[t]{2}{*}{ Stress $^{*}$} & \multicolumn{2}{|l|}{ Yes } & 4021 & 49.0 & 1117 & 48.2 & 173 & 52.4 \\
\hline & \multicolumn{2}{|l|}{ No } & 4193 & 51.0 & 1201 & 51.8 & 157 & 47.6 \\
\hline \multirow[t]{2}{*}{ Helicobacter pylori* } & \multicolumn{2}{|l|}{ Positive } & 481 & 38.5 & 307 & 39.5 & 60 & 48.8 \\
\hline & \multicolumn{2}{|l|}{ Negative } & 769 & 61.5 & 470 & 60.5 & 63 & 51.2 \\
\hline \multirow[t]{9}{*}{ Chief complaint $^{*}$} & \multirow[t]{3}{*}{ Single symptom } & & 3645 & 40.0 & 1049 & 41.2 & 131 & 36.2 \\
\hline & & epigastralgia & 1733 & 19.0 & 599 & 23.5 & 67 & 18.5 \\
\hline & & epigastric fullness & 1305 & 14.3 & 450 & 17.7 & 64 & 17.7 \\
\hline & \multirow[t]{4}{*}{ Two symptoms } & & 3328 & 36.5 & 970 & 38.1 & 151 & 41.7 \\
\hline & & heartburn/epigastralgia & 655 & 7.2 & 161 & 6.3 & 23 & 6.4 \\
\hline & & epigastralgia/epigastric fullness & 1684 & 18.5 & 518 & 20.3 & 70 & 19.3 \\
\hline & & epigastric fullness/heartburn & 989 & 10.8 & 291 & 11.4 & 58 & 16.0 \\
\hline & \multicolumn{2}{|c|}{ Three symptoms } & 2041 & 22.4 & 530 & 20.8 & 80 & 22.1 \\
\hline & & heartburn/epigastralgia/epigastric fullness & 2041 & 22.4 & 530 & 20.8 & 80 & 22.1 \\
\hline \multirow[t]{3}{*}{ Duration of chronic disease ${ }^{*}$} & \multicolumn{2}{|l|}{$<1$ month } & 4415 & 56.0 & 1258 & 56.9 & - & - \\
\hline & \multicolumn{2}{|c|}{$\geq 1$ month $<6$ months } & 2023 & 25.6 & 589 & 26.7 & - & - \\
\hline & \multicolumn{2}{|l|}{$\geq 6$ months } & 1450 & 18.4 & 362 & 16.4 & 362 & 100 \\
\hline Izumo scale (QOL) * & $\leq 2$ points (Slight) & bothered) & 2393 & 26.2 & 697 & 27.4 & 90 & 24.9 \\
\hline & $\geq 3$ points (Bother & ed) & 6730 & 73.8 & 1851 & 72.6 & 272 & 75.1 \\
\hline
\end{tabular}

*Subjects without each background factor measured were excluded.
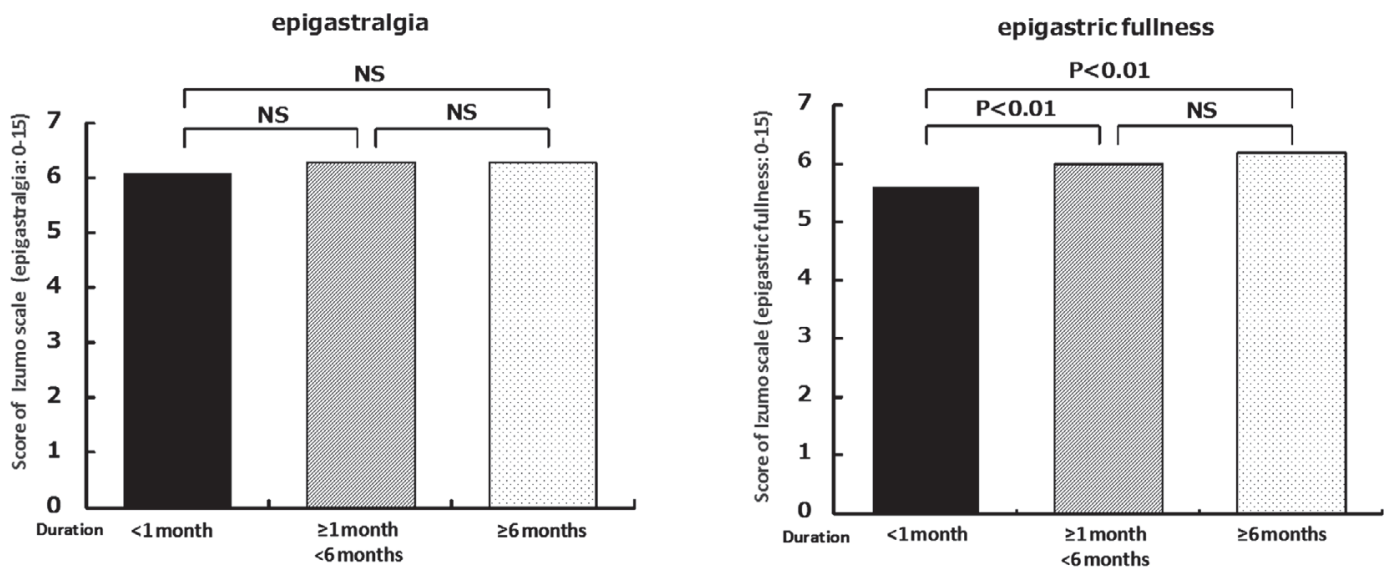

Figure 2. Abdominal symptom-related QOL impairment using Izumo scale. Duration of symptom did not strongly influence the grade of QOL impairment. NS: not significant

sicians and begin undergoing diagnostic procedures and treatment without much delay. An endoscopic examination is easily performed for dyspeptic patients in Japan (21). Abdominal symptom-related QOL is remarkably depressed as early as 1 month after the appearance of symptoms. Therefore, after excluding organic diseases by an endoscopic examination, dyspeptic patients, even if their symptom duration is shorter, should be treated similar to those with FD.

In the present study, approximately $60 \%$ of the patients with chronic gastritis as well as those with FD had more than 2 types of abdominal symptoms, which has also been reported in western countries. A study in Canada reported that over $80 \%$ of patients with dyspepsia had multiple symptoms (22). In addition, in the present study, patients with multiple symptoms were found to have lower QOL than those with a single symptom. Previously, we reported that patients with multiple abdominal symptoms more frequently consult physicians than those with a single abdomi- 

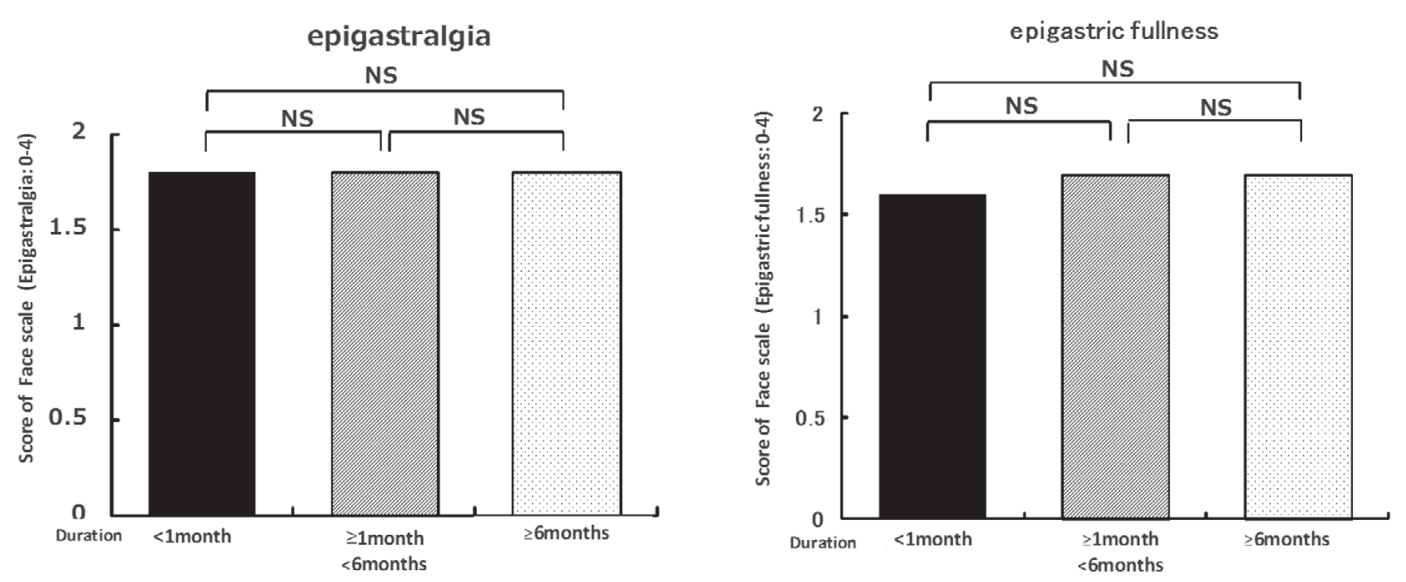

Figure 3. The severity of epigastralgia and epigastric fullness measured by a face scale. Duration of symptom did not influence the symptom severity. NS: not significant

CG patients $\square$ FD patients (A) Patients with heartburn
as chief complain

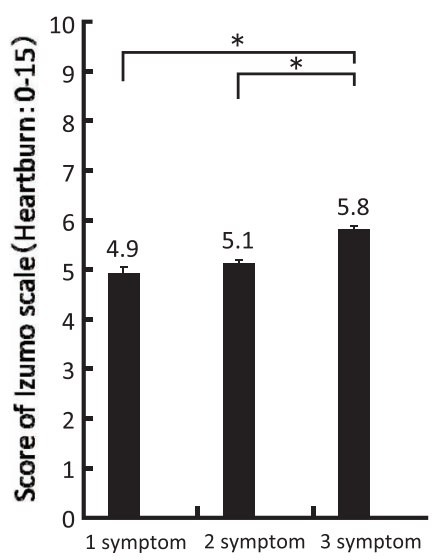

(B) Patients with epigastralgia as chief complain

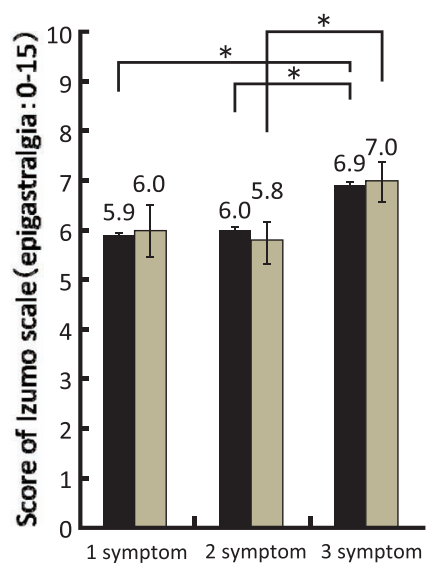

(C) Patients with epigastric fullness as chief complain

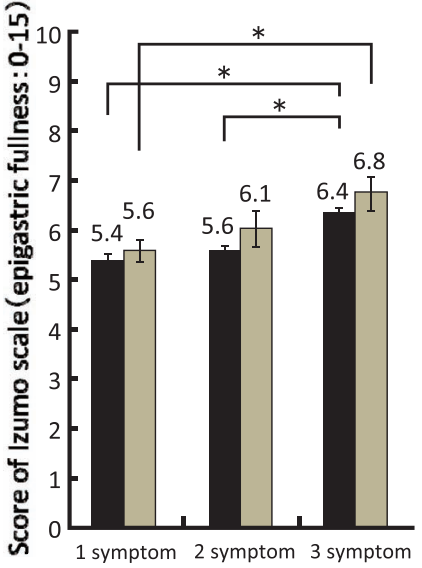

Figure 4. Abdominal symptom-related impairment of QOL measured by Izumo scale. $\square$ shows Izumo scale scores in CG patients and $\square$ shows scores in FD patients. Heartburn (A), epigastralgia (B), and epigastric fullness (C)-related QOL impairments were separately shown in patients with heartburn, epigastralgia, and epigastric fullness as their chief complaint. Patients with multiple symptoms had severe impairment of abdominal symptom specific QOL. *p<0.05 Vertical lines indicate $\mathbf{S . E}$

nal symptom. The lower QOL observed in patients with multiple symptoms may lead them to readily seek medical services. Because of their lower QOL, these patients should be treated appropriately, even if their individual symptoms may not be considered serious.

The clinical characteristics and QOL impairment of patients with chronic gastritis and ROME III defined-FD were found to be similar in the present study, with the only main difference being symptom duration. For the treatment of FD, H. pylori eradication (23), inhibitors of gastric acid secretion including $\mathrm{H}_{2} \mathrm{RA}(24-26)$ and PPI $(27,28)$, and prokinetic (29-32) and anti-anxiety (33) drugs have been reported to be effective in at least some patients. Because of the similarities between FD and chronic gastritis, treatment that is effective for FD may be investigated as a possible treatment for patients with chronic gastritis, after exclusion of organic diseases by an endoscopic examination.

In conclusion, we found similarities in the clinical characteristics including QOL impairment between patients with chronic gastritis and those with FD. Appropriate indications of an endoscopic examination and therapeutic strategy for patients with chronic gastritis are needed.

Author's disclosure of potential Conflicts of Interest (COI).

Yoshikazu K: Honoraria, Astellas Pharm Inc; Research funding, Astellas Pharm Inc. Tsutomu C: Research funding, Astellas Pharm Inc. 


\section{Acknowledgement}

This study was performed as a post-market surveillance of famotidine and supported by Astellas Pharmaceutical Co., Ltd., Tokyo.

\section{References}

1. Talley NJ, Stanghellini V, Heading RC, et al. Functional gastroduodenal disorders. Gut 45 Suppl 2: II37-II42, 1999.

2. Tack J, Talley NJ, Camilleri M, et al. Functional Gastroduodental Disorders. In: The Functional Gastrointentinal Disorders Third Edition. Printed by Allen Press Inc., Printed in the United States of America, 2006: 419-486.

3. Tack J, Tally NJ, Camilleri M, et al. Functional gastroduodenal disorders. Gastroenterology 130: 1466-1479, 2006.

4. Tack J, Tally NJ. Gastroduodenal disorders. Am J Gastroenterol 105: 757-763, 2010.

5. Camilleri M, Dubois D, Coulie B, et al. Prevalence and socioeconomic impact of upper gastrointestinal disorders in the United States: results of the US Upper Gastrointestinal Study. Clin Gastroenterol Hepatol 3: 543-552, 2005.

6. Kaji M, Fujiwara Y, Shiba M, et al. Prevalence of overlaps between GERD, FD and IBS and impact on health-related quality of life. J Gastroenterol Hepatol 25: 1151-1156, 2010.

7. Locke GR, Zinsmeister AR, Fett SL, et al. Overlap of gastrointestinal symptom complexes in a US community. Neurogastroenterol Motil 17: 29-34, 2005.

8. Manabe N, Haruma K, Hata J, et al. Clinical characteristics of Japanese dyspepsia patients: is the Rome III classification applicable? Scand J Gastroenterol 45: 567-572, 2010.

9. Furuta K, Ishihara S, Sato S, et al. Development and verification of the Izumo Scale, new questionnaire for quality of life assessment of patients with gastrointestinal symptoms. Nippon Shokakibyo Gakkai Zasshi (Japanese Journal of Gastroenterology) 106: 1478-1487, 2009 (in Japanese).

10. Furuta $K$, Ishihara $S$, Sato $S$, et al. Investigation of the responsiveness to a change in symptoms in the "Izumo scale," a questionnaire that assesses QOL in patients with gastrointestinal symptoms. Therapeutic Research 30: 1651-1657, 2009 (in Japanese).

11. Kinoshita Y. Investigation of the usefulness of the "Izumo scale," a QOL questionnaire on gastrointestinal symptoms. J New Rem \& Clin 59: 192-201, 2010 (in Japanese).

12. Kakuta E, et al. Abdominal symptom-related QOL in individuals visiting an outpatient clinic and those attending an annual health check. Intern Med 50: 1517-1522, 2011.

13. Armstrong D, Bennett JR, Blum AL, et al. The endoscopic assessment of esophagitis: a progress report on observer agreement. Gastroenterology 111: 85-92, 1996.

14. Shiota S, Murakami K. Endoscopic findings of dyspeptic patients. Clinical Gastroenterology 51: 467-470, 2010.

15. Talley NJ, Vakil NB, Moayyedi $P$, et al. American gastroenterological association technical review on the evaluation of dyspepsia. Gastroenterology 129: 1756-1780, 2005.

16. Taha AS, McCloskey C, Prasad R, et al. Famotidine for the prevention of peptic ulcers and oesophagitis in patients taking lowdose aspirin (FAMOUS): a phase III, randomised, double-blind, placebo-controlled trial. Lancet 374: 119-125, 2009.

17. Bhatt DL, Scheiman J, Abraham NS, et al. ACCF/ACG/AHA 2008 expert consensus document on reducing the gastrointestinal risks of antiplatelet therapy and NSAID use. Am J Gastroenterol 103: 2890-2907, 2008.

18. Ootani H, Iwakiri R, Shimoda R, et al. Role of Helicobacter py- lori infection and nonsteroidal anti-inflammatroy drug use in bleeding peptic ulcers in Japan. J Gastroenterol 41: 41-46, 2006.

19. Sakamoto C, Sugano K, Ota S, et al. Case-control study on the association of upper gastrointestinal bleeding and nonsteroidal anti-inflammatory drugs in Japan Eur. J Clin Pharmacol 62: 765772,2006

20. Kamata T, Yoneda M, Kawamoto $H$, et al. A study of gastric cancer mass screening using the serum pepsinogen test in combination with the barium X-ray method in Maniwa City, Okayama Prefecture. J Gastroenterological Cancer Screening 45: 514-521, 2007.

21. Kitagawa S, Miyagawa K, Utsunomiya $S$, et al. Gastroenterological Cancer Screening Report in 2008. The Japanese Society of Gastroenterological Cancer Screening Committee Report. J Gastroenterological Cancer Screening 49: 73-98, 2011.

22. Thomson ABR, Barkun AN, Armstrong D, et al. The prevalence of clinically significant endoscopic findings in primary care patients with uninvestigated dyspepsia: the Canadian Adult Dyspepsia Empiric Treatment-Prompt Endoscopy (CADET-PE) study. Aliment Pharmacol Ther 17: 1481-1491, 2003.

23. Kamada T, Haruma K, Hata J, et al. The long-term effect of Helicobacter pylori eradication therapy on symptoms in dyspeptic patients with fundic atrophic gastritis. Aliment Pharmacol Ther 18: 245-252, 2003.

24. Kato M, Watanabe M, Konishi S, et al. Randomized, double-blind, placebo-controlled crossover trial of famotidine in patients with functional dyspepsia. Aliment Pharmacol Ther 21 (Suppl. 2): 2731, 2005.

25. Seno H, Nakase H, Chiba T. Usefulness of famotidine in functional dyspepsia patient treatment: comparison among prokinetic, acid suppression and antianxiety therapies. Aliment Pharmacol Ther 21 (Suppl. 2): 32-36, 2005.

26. Kinoshita K, Hashimoto T, Kawamura A. Effects of famotidine, mosapride and tandospirone for treatment of functional dyspepsia. Aliment Pharmacol Ther 21 (Suppl. 2): 37-41, 2005.

27. Meineche-Schmidt V, Christensen E, Bytzer P. Randomised clinical trial: identification of responders to short-term treatment with esomeprazole for dyspepsia in primary care-a randomised, placebo-controlled study. Aliment Pharmacol Ther 33: 41-49, 2011.

28. Peura DA, Kovacs TO, Metz DC, et al. Lansoprazole in the treatment of functional dyspepsia: two double-blind, randomized, placebo-controlled trials. Am J Med 116: 740-748, 2004.

29. Matsueda $K$, Hongo $M$, Tack $J$, et al. Clinical trial: dosedependent therapeutic efficacy of acotiamide hydrochloride (Z338 ) in patients with functional dyspepsia $-100 \mathrm{mg}$ t.i.d. is an optimal dosage. Neurogastroenterol Motil 22: 618-625, 2010.

30. Tack J, Masclee A, Heading R, et al. A dose-ranging, placebocontrolled, pilot trial of Acotiamide in patients with functional dyspepsia. Neurogastroenterol Motil 21: 272-280, 2009.

31. Hiyama T, Yoshihara M, Matsuo K, et al. Treatment of functional dyspepsia with serotonin agonists: a meta-analysis of randomized controlled trials. J Gastroentero Hepato 22: 1566-1570, 2007.

32. Holtmann G, Talley NJ, Liebregts T, et al. A placebo-controlled trial of itopride in functional dyspepsia. N Engl J Med 354: 832840, 2006.

33. Miwa H, Tomaga K, Yokoyama T, et al. Efficacy of the 5-HT1A agonist tandospirone citrate in improving symptoms of patients with functional dyspepsia: a randomized controlled trial. Am J Gastroenterol 104: 2779-2787, 2009.

(C) 2011 The Japanese Society of Internal Medicine http://www.naika.or.jp/imindex.html 